\title{
Telemetric ECG Diagnosis Follow-Up
}

\author{
R Bousseljot, U Grieger, D Kreiseler, L Schmitz, H Koch, S Beckmann*, C Bethge**, \\ $\mathrm{H}$ von Nettelhorst $\uparrow$, K Stangl†
}

\author{
Physikalisch-Technische Bundesanstalt, Berlin, Germany \\ *Clinic for Rehabilitation Cardiology, Rankestrasse, Berlin, Germany \\ **Jüdisches Krankenhaus, Berlin, Germany \\ $\dagger$ Getemed Inc., Teltow, Germany \\ †Charité Medical Center, Humboldt University, Berlin, Germany
}

\begin{abstract}
A telemetric tool for the evaluation and re-evaluation of a series of digitized 12-lead ECG samples from an individual patient was developed. The solution involves a standard PC-based client/server concept with communication via Internet. The ECG is recorded on a commercial Web-linked PC-based device and the pre-processed data are transmitted to the server together with a set of additional information. A cross-correlation algorithm is then used to compare the ECGs to a large validated ECG database. The likelihood of diagnoses is assigned to each ECG, and changes in the ECG followup can be demonstrated in a comprehensive series of graphs. This new tool, by providing the total ECG history of an individual patient to any authorized health professional, improves clinical judgement, and may significantly improve cost-effectiveness in health-care practice when it becomes widely used.
\end{abstract}

\section{Introduction}

The evaluation of an ECG follow-up is one of the most commonly performed basic procedures in the care of patients with significant heart diseases. As the individual patient's ECGs are most likely taken at different institutions rather than at a single location, the follow-up is usually based on the descriptions and interpretations of ECGs by the involved physicians, rather than on a post hoc re-evaluation of the collected ECG strips by health professionals involved in decision-making in a given clinical situation. Therefore, a technical tool enabling data storage, automated ECG interpretation, and trend analysis was developed.

\section{Concept of ECG follow-up analysis}

The backbone of the follow-up analysis is a pattern comparison method for computerized 12-lead ECG interpretation that has been described in detail in $[1,2]$. The digitized ECG obtained from a patient is compared to 24,000 ECGs of a validated database and a histogram is constructed as shown in Fig. 1 from the associated diagnoses of the 50 database ECGs matching best the ECG under question. This histogram contains the following diagnostic classes: normal (NORM), myocardial infarction (MI), ischemia (ISC), ventricular hypertrophy $(\mathrm{VH})$, and bundle branch block (BBB). With the exception of class NORM, each other class can be sub-classified into the location of the event, and in addition, for the class MI into the age of the event. Thus, such a histogram not only allows the assignment of the most probable diagnosis class(es) to the new ECG but also provides a quantitative measure of the probability for a valid assignment and on the probabilities for an alternative and/or compound diagnosis.

Knowing these probabilities is already a value in itself but - in addition - it allows forming the basis of a quantitative follow-up analysis: a comparison of such histograms at subsequent instants of patient monitoring directly provides a visualization of trends of the cardiac status of the patient. This concept has been adapted to a practical solution involving a prototype network of client/server-PCs between three clinics (see affiliation of authors), an ECG device manufacturer (getemed) and a research and development center (PTB) connected via Internet. 


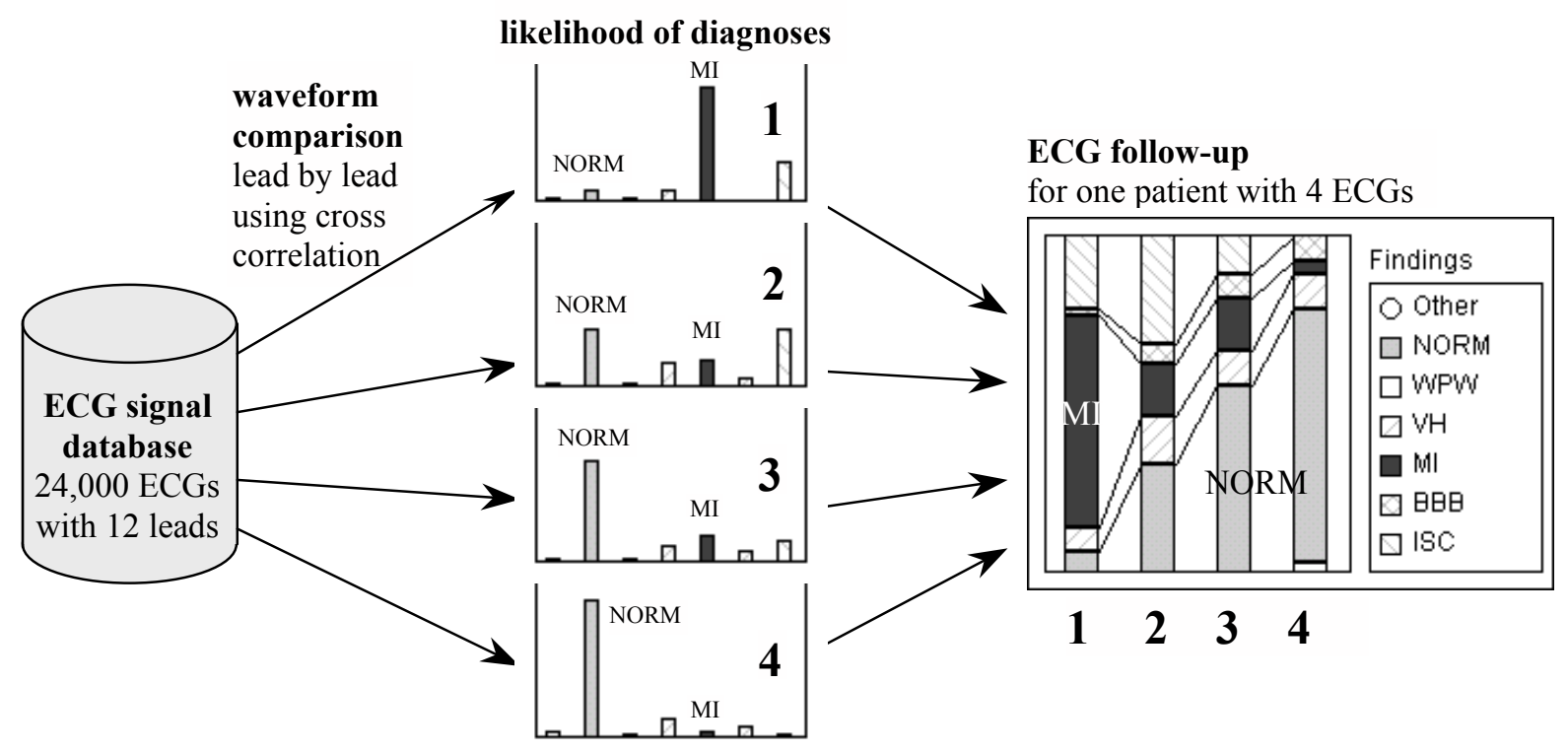

Figure 1. Principle of the ECG follow-up analysis.

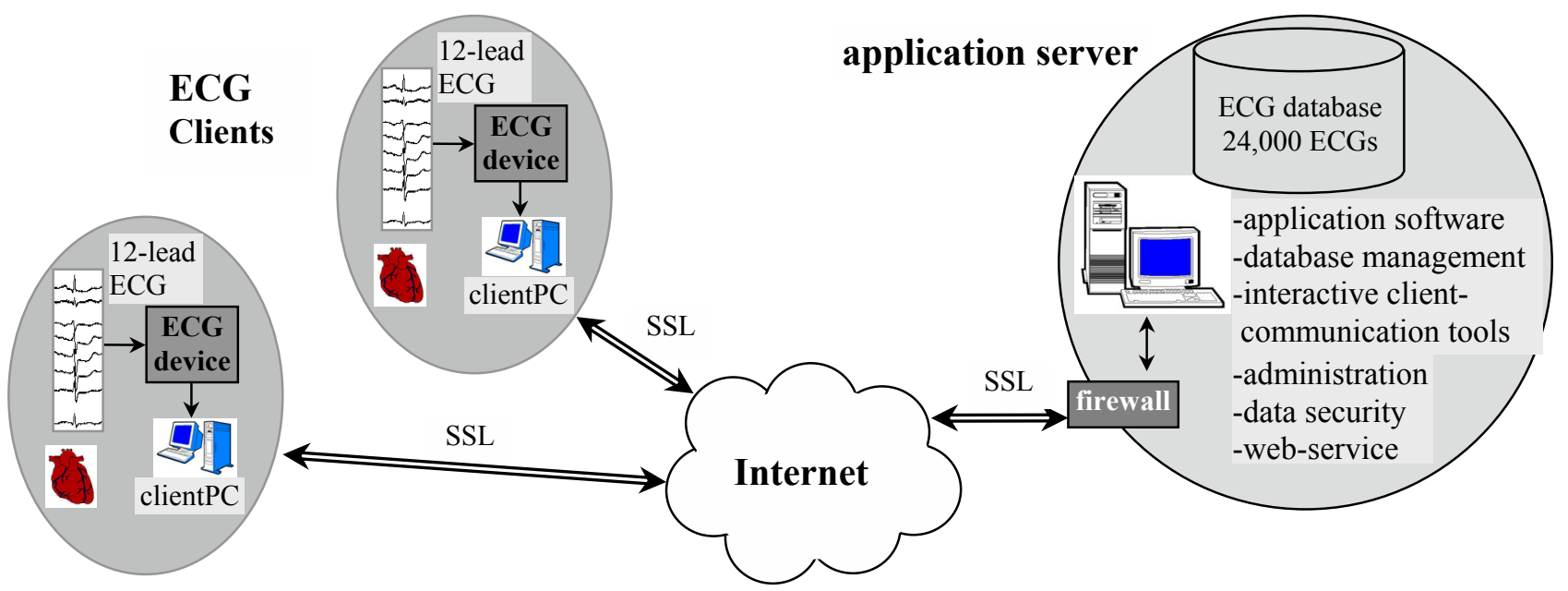

Figure 2. ECG pattern identification on an application server with two connections via Internet.

\section{Prototype solution}

Modular software variants for PC were developed in order to implement the method (Figure 2). Structuring of the problem led to three program packages that have to be executed one after the other. The programs can be stored on different computers.

Provision of the 12-lead ECG from the electrocardiograph as well as the required signal conditioning and beat selection are typically performed by the peripherals at the place where the ECG is recorded (client's computer). The comparison of the patterns with the database ECGs is the task of a central computer (application server).

Special conditioning of the signal segments allows the comparison between the reference ECG and the ECGs stored in the database to be carried out on a PC within less than 2 minutes. The presentation of the results and interactions between users and database takes place again on the client's computer. 


\section{Results}

\subsection{CSE evaluation}

The performance of the ECG interpretation algorithm used in this study has been evaluated by the group of Prof. Rubel, Lyon, France, using the CSE (Common Standards for Quantitative Electrocardiography) diagnostic library data stet 5 (1,220 ECGs). The results are summarized in the table.

Table: 1. Comparison with the Clinical Truth CSEprogram DIBV

\begin{tabular}{lcc}
\hline & $\begin{array}{c}\text { Sensitivity } \\
\%\end{array}$ & $\begin{array}{c}\text { Specificity } \\
\%\end{array}$ \\
\hline Normal & 85.9 & 85 \\
MI & 75.2 & 81 \\
VH & 42.8 & 94.3 \\
\hline Total accuracy & 73.4 & \\
\hline
\end{tabular}

At a first shot, the diagnostic performance of the applied waveform comparison algorithm was within the range of other deterministic programs that have been evaluated by the CSE program [3].

\subsection{Follow-up}

The performance of the ECG follow-up algorithm is demonstrated by the following case reports. They are selected from a library of 210 ECGs from 48 patients.

Case 1: \#42, male, 50 years, transmural anteroseptal myocardial infarction (Q-wave MI), 3-vessel disease. Measurements: on admission, day 5, day 11 , and 22 months after the infarction.

Case 2: \#48, male, 64 years, anteroseptal myocardial infarction (Q-wave MI), persistent scar, 2-vessel disease. Measurements: on admission, day 3, day 11, and 11 months after the infarction.

Case 3: \#83, male, 45 years, discrete anteroseptal myocardial infarction (non-Q-wave MI) without dyskinesia, single vessel disease. Measurements: on admission, day 3 , day 7 , and day 12 .

Case 4: \#69, male, 62 years, extended anteroseptal and lateral myocardial infarction (non-Q-wave MI), single vessel disease. Measurements: on admission, day 3, day 10 , and 5 months after the infarction.

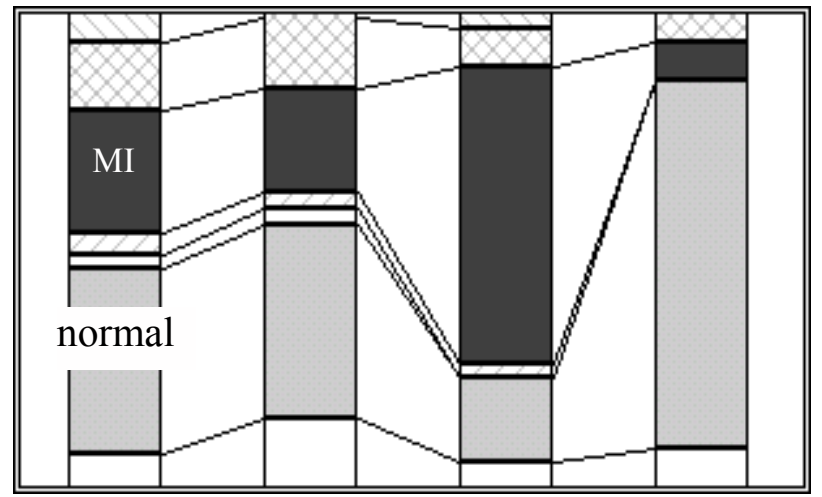

Figure 3. \#42, typical healing of a Q-wave myocardial infarction (MI).

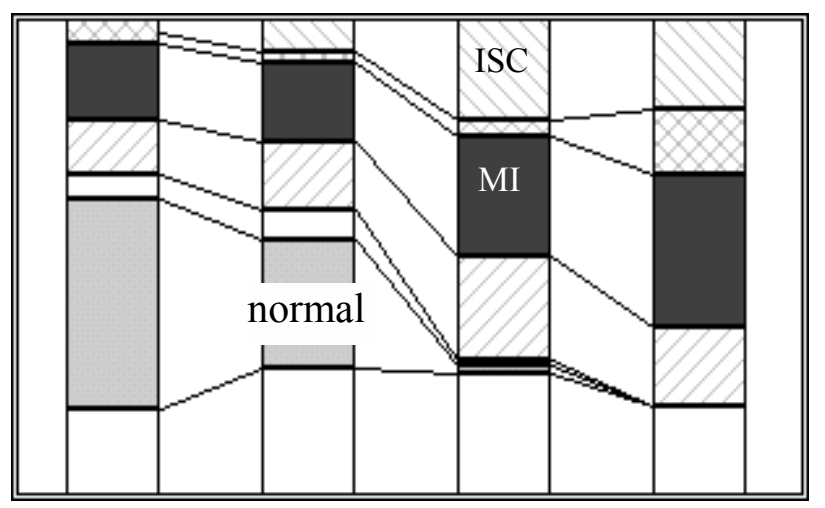

Figure 4. \#48, infarction (MI) with persistent ECG scar and/or persistent ischemia (ISC).

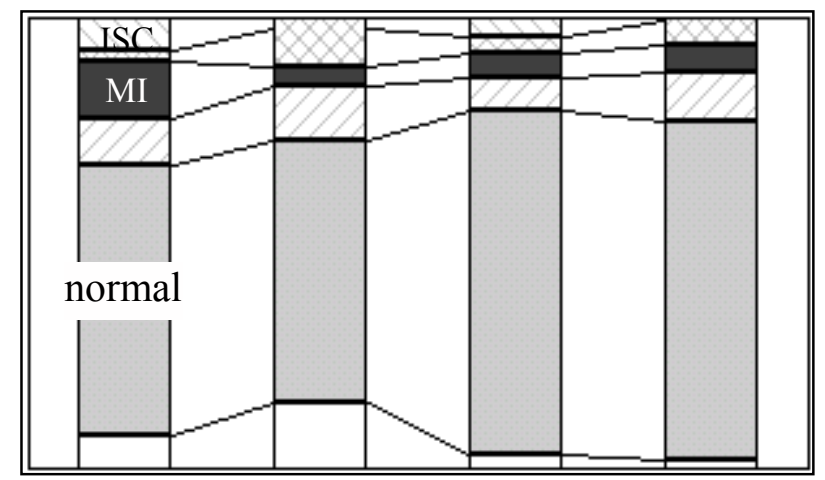

Figure 5. \#83, small localized infarction with only discrete increases in the infarction (MI) and/or ischemia (ISC) pattern. 


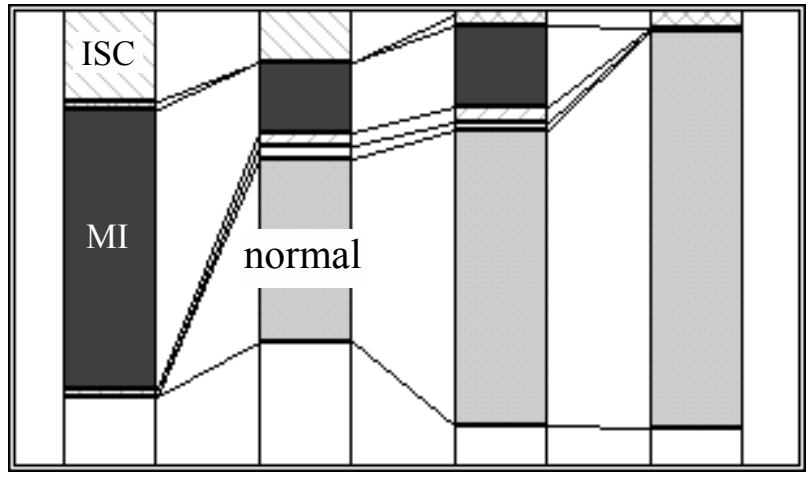

Figure 6. \#69, non-Q-wave infarction shows the typical infarction (MI) plus ischemia (ISC) pattern.

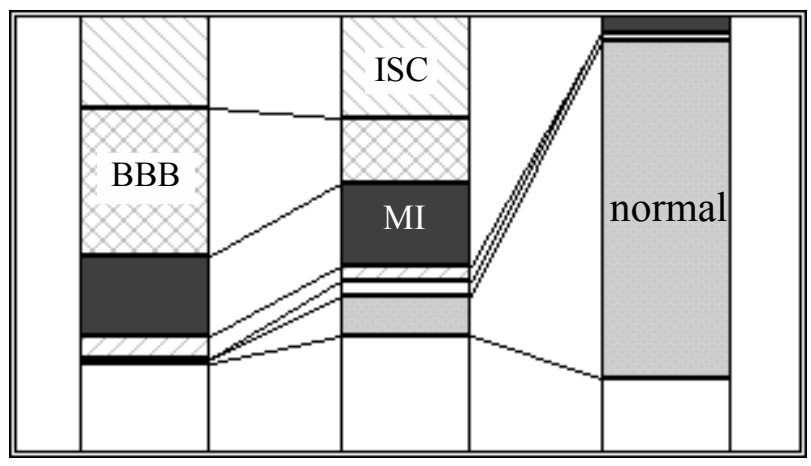

Figure 7. \#67; true posterior infarction characterized by equally increased infarction (MI) and ischemia (ISC) patterns. Transient left anterior fascicular block (BBB).

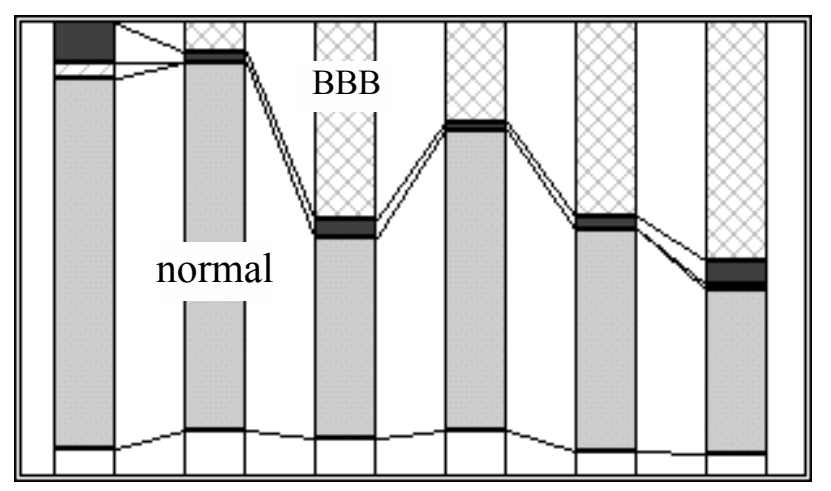

Figure 8. \#284, classification shows the development of a bundle branch block (BBB). Sub-classification (not displayed) showed the absence of bundle branch block in measurements 1 and 2, incomplete right bundle branch block in measurements 3 and 4, and complete right bundle branch block in measurement 5 and 6 .
Case 5: \#67, male, 63 years, posterior myocardial infarction and intermittent block (left anterior fascicular block) at first exam, 3-vessel disease. Measurements: on admission, day 35, and 6 months after the infarction.

Case 6: \#284. male, 47 to 50 years, developing block over 4 years. No other cardiac abnormalities.

\section{Summary and outlook}

As could be demonstrated, the application for ECG follow-up yields comprehensive results. The tool is now under further evaluation in a clinical routine setting. Preliminary results show that for patients with chronic heart diseases and persisting ECG scars, this new method substantially enhances the diagnostic power of the ECG follow-up procedure.

Furthermore, this telemetric solution offers the possibility of having the patient's complete ECG library on the screen of every doctor or paramedical staff member caring for the patient wherever the involved persons happen to be. Besides from facilitating clinical decisionmaking, this telemetric solution might importantly improve cost-effectiveness in health-care practice when it becomes widely used.

Additional information may be obtained at http:// www.berlin.ptb.de/8/82/821/ecg/index.html. The introduced method of ECG follow-up is covered by patents.

\section{Acknowledgements}

The support by BMBF-grant \# FKZ 01AK051 is highly appreciated.

\section{References}

[1] Bousseljot R, Kreiseler D. Waveform recognition with 10,000 ECGs. Computers in Cardiology 2000. Piscataway, NJ: IEEE, 2000:331-4.

[2] Bousseljot R, Kreiseler D. Results of ECG interpretation by means of signal wave recognition. Herzschr Elektrophys 2000; 197-06.

[3] Willems JL, et al. The diagnostic performance of computer programs for the interpretation of electrocardiograms. N Engl J Med 1991;325:1767-73.

Address for correspondence:

Prof. Dr. Hans Koch

Physikalisch-Technische Bundesanstalt

Abbestrasse 2-12

D-10587 Berlin, Germany

e-mail: hans.koch@ptb.de 